\title{
$\$$ Research Square

\section{Interface Modeling for the Ab Initio Evaluation of the Water Contact Angle on a Metallic Cu(111) Surface}

\author{
Takahiro Murono \\ JAIST \\ Kenta Hongo ( $\nabla$ kenta_hongo@mac.com ) \\ JAIST
}

Kousuke Nakano

JAIST

Ryo Maezono

JAIST

\section{Research Article}

Keywords: Interface Modeling, Ab Initio Evaluation, Water Contact Angle, Metallic Cu(111) Surface

Posted Date: July 15th, 2021

DOl: https://doi.org/10.21203/rs.3.rs-706533/v1

License: (c) (i) This work is licensed under a Creative Commons Attribution 4.0 International License.

Read Full License 


\title{
Interface Modeling for the $A b$ Initio Evaluation of the Water Contact Angle on a Metallic Cu(111) Surface
}

\author{
Takahiro Murono $^{1}$, Kenta Hongo ${ }^{2}$, Kousuke Nakano ${ }^{1,3}$, and Ryo Maezono ${ }^{1}$ \\ 1 School of Information Science, JAIST, \\ Asahidai 1-1, Nomi, Ishikawa, 923-1292, Japan \\ ${ }^{2}$ Research Center for Advanced Computing Infrastructure, \\ JAIST, Asahidai 1-1, Nomi, Ishikawa 923-1292, Japan and \\ ${ }^{3}$ International School for Advanced Studies (SISSA), \\ Via Bonomea 265, 34136 Trieste, Italy
}

\begin{abstract}
Controlling the water contact angle on a surface is important for regulating its wettability in industrial applications. Therefore, it is crucial to develop ab initio evaluation methods that can accurately predict this angle. The $a b$ initio predictions require an adsorption structure model for the adsorption of liquid molecules on a surface, but the construction of this model depends on whether the test surface comprises an insulating or metallic material because the surface reconstruction takes quite a different form in each case. Previous studies have focused on the estimation of the water contact angle on insulators; however, this study elucidates the water contact angle on a metallic surface, $\mathrm{Cu}(111)$. Because the feasibility of ab initio evaluations depends on the approximation of liquid-gas interface energy, which can be roughly estimated through the interface energy of crystal ice, it is natural to use the periodic-honeycomb array of water molecules as the adsorption model for the water on the surface. However, despite the successful application of the periodic model for $a b$ initio prediction of the water contact angle on insulating surfaces, applying this model to metallic surfaces has not provided satisfactory predictions that reproduce experimental values. Therefore, in this study, we propose the use of models with isolated water oligomers for the ab initio prediction of the water contact angle on a metallic surface, which achieved an accurate prediction. The ambiguity of the models based on the size and coverage of the oligomers was small $\left(\sim \pm 10^{\circ}\right)$, which was averaged out to give a plausible value based on the Boltzmann weight with the adsorbing energies. The proposed procedure can be used to estimate the wettability of the surfaces of other metallic materials.
\end{abstract}




\section{INTRODUCTION}

Regulating the wettability of surfaces [1-3] is an important issue that must be addressed to broaden their industrial applications, such as heterogeneous catalysis, corrosion, and electrochemistry. $[1,4,5]$ The wettability of the surfaces of metal catalysts such as $\mathrm{Cu}$ has a significant effect on their catalytic activity for electrochemical reactions, [6] wherein the receding contact angle of water on the metal surface corresponds to the oxygen reduction reactions of the catalyst. This indicates that the efficiencies of the electrochemical reactions of metal catalysts can be enhanced by controlling the water contact angle with the metal surface.

The contact angle $(\theta)$ is the primary measure of wettability; a larger contact angle $\left(\theta>90^{\circ}\right)$ indicates hydrophobicity, i.e., low wettability, whereas a smaller contact angle $\left(0^{\circ}<\theta<90^{\circ}\right)$ indicates hydrophilicity, i.e., high wettability. Experimentally, the water contact angle on metal surfaces can be measured directly by observing the angle captured by the cameras. [7, 8] The angle is determined using the Young's relation $[9,10]$ :

$$
\cos \theta=\frac{\gamma_{\mathrm{SG}}-\gamma_{\mathrm{SL}}}{\gamma_{\mathrm{LG}}},
$$

where $\gamma_{\mathrm{SG}}, \gamma_{\mathrm{LG}}$, and $\gamma_{\mathrm{SL}}$ are the surface energies at solid-gas, liquid-gas, and solid-liquid interfaces, respectively. Although these energies can be computed by various approaches, the proposed study focuses on $a b$ initio evaluations (the comparison with other approaches is provided later). The $a b$ initio prediction of water contact angles on surfaces was recently introduced, and it has been successfully applied to estimate this angle on the surfaces of insulators. $[1,11]$ For example, the contact angle of water on Si [11] was predicted via ab initio evaluation $\left(88^{\circ}\right)$, which was close to the experimental value $\left(91^{\circ}\right)$. In addition, ab initio evaluations have also been applied to estimate the water contact angle on transition metal oxides [1]. For example, the water contact angle on $\mathrm{CeO}_{2}(111)\left[\mathrm{Nd}_{2} \mathrm{O}_{3}(0001)\right]$ was estimated via ab initio evaluations as $100^{\circ}\left[103^{\circ}\right]$, which is comparable to the experimental value, i.e., $103^{\circ} \pm 2^{\circ}\left[101^{\circ} \pm 3^{\circ}\right]$; this result indicates that the $a b$ initio evaluations are in accordance with actual values. For the estimations performed in previously reported studies, the $\gamma_{\mathrm{LG}}$ of water was substituted with that of crystal ice because water and crystal ice have similar surface energies [12].

Previously reported studies based on the $a b$ initio evaluations of contact angles of water at different surfaces have considered insulators as the surfaces. In previous studies, $[1,11]$ an "ice-like bilayer model" $[13,14]$ (periodic-honeycomb structure of ice) has been adopted as the adsorption model for conducting the ab initio evaluations of the contact angles of water on various insulator 
surfaces, which is a natural choice for the $\gamma_{\mathrm{LG}}=\gamma_{\text {ice }}$ model (see Fig.1 (a)). However, when estimating the water contact angle on the surface of metals, the structural modeling to describe the adsorption of water molecules on the metallic surface should be different from that used to describe the adsorption of water molecules on the insulator surface. Previous studies have reported the presence of isolated water hexamers on the surface of metals such as $\mathrm{Cu}[15,16]$, indicating that the "bilayer model" is not appropriate for the ab initio evaluation of the water contact angle on the metal surface. Therefore, "isolated models" that do not exhibit the bridging of hydrogen bonds over neighboring unit cells would be more appropriate.

In this study, we construct an isolated model for conducting the $a b$ initio evaluations of the water contact angle on a metallic surface. First, we investigated the advantages offered by the modeling method. We observed that the type of $N$-mer/oligomer (ranging from monomer to hexamer) and number of $N$-mers within a unit cell should be specified. Particularly, we examined the dependence of the $a b$ initio prediction on the specific modeling choice. We confirmed that (i) the contact angle of water molecules on the metallic $\mathrm{Cu}(111)$ surface, predicted using the ab initio evaluations with isolated modeling, was closer to the experimental value than that obtained with the periodic honeycomb modeling. Further, (ii) the freedom of selection $[(N$-mer $) \otimes($ coverage $)]$ and the averaging with the Boltzmann weight based on the adsorption energy were useful.

The paper is organized as follows. The "Computational Methods" is divided into two subsections. The "Interface modeling" subsection provides a detailed description of our "isolated cluster" models. The "Computational details" subsection specifies our ab initio methods of evaluating the energies based on the models. The "Results and Discussion" section shows that the numerical results are comparable with available experiment results, and deals with pros and cons of our approach by comparing the proposed approach with other approaches. Finally, the findings are summarized in the "Conclusion" section.

\section{COMPUTATIONAL METHODS}

\section{A. Interface modeling}

Based on the previous studies, $[1,11]$ we estimated

$$
\gamma_{\mathrm{SG}}-\gamma_{\mathrm{SL}}=-E_{\mathrm{ads}}^{\mathrm{water}} / A
$$


(a)

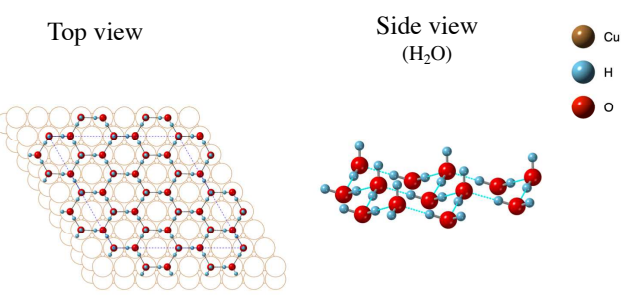

(b)

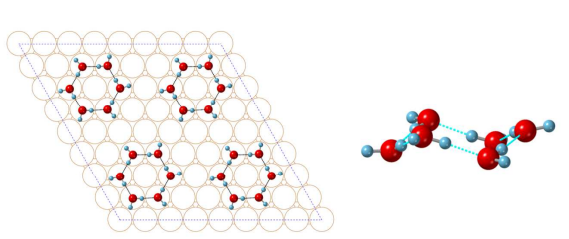

(b’)

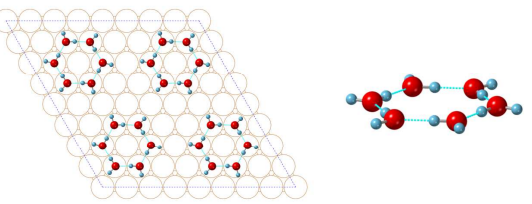

FIG. 1. Structural models describing the adsorption of water on metal surface for the ab initio evaluation of the contact angles. Periodic honeycomb structure known as the "bilayer model." [13, 14] Panel (a) shows the bilayer model, which is used for the estimation of the contact angles of water on insulator surfaces. [1, 11] Panel (b) and (b') show the "isolated molecular (cluster) models," which were found to be more appropriate for the estimation of the water contact angle on metal surfaces.

where $E_{\mathrm{ads}}^{\mathrm{water}}$ is the adsorption energy of water molecules on the surface ( $A$ is the unit area within which $E_{\text {ads }}^{\text {water }}$ is defined). For the liquid-gas interfaces,

$$
\gamma_{\mathrm{LG}}=\gamma_{\mathrm{ice}},
$$

which was evaluated using the surface energy of ice, $\gamma_{\text {ice }}[1,11]$ Then, the formula for the contact angle can be provided as follows:

$$
\cos \theta=\frac{-E_{\text {ads }}^{\mathrm{water}}}{A \cdot \gamma_{\mathrm{ice}}}
$$

which can be reduced to the ab initio energy evaluations. $E_{\text {ads }}^{\text {water }}$ is given as [1]:

$$
E_{\text {ads }}^{\text {water }}=E_{\text {tot }}-E_{\text {water }}(\text { onSurf })-E_{\text {CuSlab }},
$$


where $E_{\text {CuSlab }}, E_{\text {water }}$ (onSurf), and $E_{\text {tot }}$, are the energy of the Cu slab, energy of water molecules on the surface of the adsorbed structure, and total energy of the system with the molecules on the surface, respectively. The surface energy $\gamma_{\text {ice }}$ can be evaluated as:

$$
\gamma_{\text {ice }}=\frac{E_{\text {ice }}(n ; \text { Slab })-E_{\text {ice }}(n ; \text { Bulk })}{2 A_{\text {ice }}}
$$

which is a measure of the stabilization of the ice surface, where $E_{\text {ice }}(n ;$ Slab $)$ and $E_{\text {ice }}(n ;$ Bulk $)$ are the energies of the slab and the bulk of the ice composed of $n$ water molecules, respectively.

The water contact angle on the surface is estimated using five quantities: $E_{\text {ice }}(n ;$ Bulk), $E_{\text {ice }}\left(n ;\right.$ Slab), $E_{\text {CuSlab }}, E_{\text {water }}\left(\right.$ onSurf), and $E_{\text {tot }}$, which can be evaluated using $a b$ initio density functional theory (DFT) calculations. Considering $\mathrm{Cu}(111)$ as the surface, we calculated the energies using a DFT package, CASTEP. [17] For the ease of comparison with previous works, [1, 11] we used the same exchange-correlation function, GGA-PBE, [18] used in previous studies. In addition, norm-conserving pseudopotentials [19] were used to describe the ionic cores. The detailed computational conditions for each calculation is summarized in Table I.

The detailed information used for modeling the geometries of the adsorbed molecules is provided in the subsequent subsection: Computational Details. The main comparison was conducted between the predictions achieved by the periodic-honeycomb model (bilayer) [13, 14] [panel (a) in Fig. 1] and those by the isolated molecular models (buckled) [16, 20] [panel (b)], which were evaluated using a $\mathrm{Cu}$ slab comprising nine atomic layers. Furthermore, to investigate the existence of the considerable bias on the choice of $N$-mer and coverage, we compared the predictions with $N=1,2,3,4$, and 6; however, this comparison was conducted with reduced cost and complexity of the computation, namely with an H-parallel model [21] (planar model) [panel (b') in Fig. 1] and with reduced number of layers (four). $N=5$ was excluded because we limited the possible geometry to satisfy the "on-top alignment," which is supported by the findings of previous studies $[20,21]$ [i.e., $N=5$ cannot accommodate the molecules within a unit cell, such that they have an "on-top alignment" geometry]. For the oligomers, $N>2$, they can either assume the chain or circular form. Therefore, we considered both possibilities for $N=3$ and 4 and considered only the circular form for $N=6$, as supported by previous studies. [16, 20] The dependence on the coverage, i.e., on the number of $N$-mers placed within a unit cell, was examined up to $N=3$. 
TABLE I. Computational details of the DFT calculations for each energy to evaluate the contact angles of water on the metallic $\mathrm{Cu}(111)$ surface. The $k$-mesh and $E_{\mathrm{CUT}}$ (given in $[\mathrm{eV}]$ ) are the mesh size for the Brillouin zone [22] and the plane wave energy cutoff, respectively. These values were determined by the convergence of the total energies. 'Cu-struct-opt' and 'Cu-surf-relax' indicate the optimized $\mathrm{Cu}$ bulk and $\mathrm{Cu}$-slab structures, respectively.

\begin{tabular}{lcc}
\hline & $k$-mesh & $E_{\text {CUT }}[\mathrm{eV}]$ \\
\hline$E_{\text {ice }}(n ;$ Bulk $)$ & $4 \times 2 \times 2$ & 750 \\
$E_{\text {ice }}(n ;$ Slab $)$ & $4 \times 4 \times 1$ & 750 \\
$E_{\text {CuSlab }}$ & $1 \times 1 \times 1$ & 700 \\
$E_{\text {water }}($ onSurf $)$ & $1 \times 1 \times 1$ & 600 \\
$E_{\text {tot }}$ & $1 \times 1 \times 1$ & 700 \\
\hline Cu-struct-opt & $8 \times 8 \times 8$ & 800 \\
Cu-surf-relax & $11 \times 11 \times 1$ & 800 \\
\hline
\end{tabular}

\section{Computational details}

For the energies required to evaluate the contact angles, we prepared the geometries of the $\mathrm{Cu}$ slab (for $E_{\mathrm{CuSlab}}$ ), ice bulk slab (for $E_{\mathrm{ice}}(n ; \mathrm{Bulk})$ and $E_{\mathrm{ice}}(n ; \mathrm{Slab})$ ), and the water molecules adsorbed on the $\mathrm{Cu}$ slab (for $E_{\text {water }}\left(\right.$ onSurf) and $E_{\text {tot }}$ ).

A Cu bulk with an initial lattice constant of $3.6147 \AA$ was prepared to construct the Cu-slab structure. The constant was optimized under the bulk structure as $3.728 \AA$; subsequently, the bulk was cleaved along the (111) plane and used as the initial structure of the slab. Then, a nine(four)layered slab was located periodically, on which a $30 \AA$ deep vacuum layer was attached; the atomic positions within the three (one) layers from the surface were relaxed by optimizing the geometry to obtain the final structure of the $\mathrm{Cu}$ slab. For the ice bulk, we used the Ih structure with a lattice constant of $4.516 \AA$. [23, 24] The ice bulk was cleaved to obtain four layers of $\mathrm{H}_{2} \mathrm{O}$, which were parallel to the basal plane of the Ih crystal. The $\mathrm{Cu}$ slab was formed by attaching a vacuum layer with a $30 \AA$ depth, without further relaxations.

The water molecule was prepared using the initial geometry, $l_{\mathrm{O}-\mathrm{H}}=0.96 \AA$ and $\theta_{\mathrm{H}-\mathrm{O}-\mathrm{H}}=$ $104.5^{\circ}$. For the periodic-honeycomb model [panel (a) in Fig. 1], we used the "bilayer model" employed in preceding studies. $[13,14]$ The structure was generated from the "H-parallel" struc- 
TABLE II. Predicted water contact angles on a $\mathrm{Cu}(111)$ surface using different absorption models. "Boltzmann ave." implies the average of several isolated models with different sizes of isolated molecules, providing varying coverage (see text).

\begin{tabular}{ccc}
\hline Model & Simulation size Contact angle $\left[{ }^{\circ}\right]$ \\
\hline Periodic-honeycomb (bilayer) & nine-layer & 69.34 \\
Isolated-hexamer (buckled) & nine-layer & 79.59 \\
Boltzmann ave. (planar, 297 K) & four-layer & 78.47 \\
\hline Exp. [25] & - & 86.3 \\
Exp. [26] & - & 75.26 \\
\hline
\end{tabular}

ture, [21] where all the $\mathrm{H}_{2} \mathrm{O}$ molecular planes were parallel to the slab surface. Then, the positions of the oxygen atoms were adjusted such that their heights from the slab surface were alternated in every molecule to form the buckled structure. $[16,20]$ To evaluate the contact angles in the fourlayer slab models (as shown later in Fig. 2), water molecules were placed on the models using the on-top alignment geometry [20,21] with the H-parallel orientation [21]; herein, the vertical height was optimized by DFT.

\section{RESULTS AND DISCUSSION}

As shown in Table II, the periodic-honeycomb model (bilayer) [1, 11] is the most inferior model from the viewpoint of the similarity of results to the experimental values obtained for the water contact angle on the metallic $\mathrm{Cu}(111)$ surface. $[25,26]$ In contrast, the isolated molecular models (buckled and planar) [14, 16] were more appropriate for predicting the water contact angle on metal surfaces. The difference in their prediction accuracy can be attributed to the bridging of the hydrogen bonds over neighboring unit cells. In addition, because the lattice mismatch between the $\mathrm{Cu}$ surface and honeycomb network was $-2.1 \%$ [13], the difference in the accuracy of the predictions cannot be attributed to the lattice mismatch between the $\mathrm{Cu}$ surface and honeycomb network.

As mentioned in the Introduction section, the freedom of choice, with respect to [( $N$-mer $) \otimes($ coverage $)]$ was achieved for the isolated molecular model. We examined the dependence of the predictions on various models, but with low-computational-cost (four-layer) and complex (planar) structures; the 
results are shown in Fig. 2. Consequently, the contact angle changes from $79.59^{\circ}$ (buckled/nine layers) $\rightarrow 77.13^{\circ}$ (buckled/four layers) and $\rightarrow 77.29^{\circ}$ (planar/ four -layers), which is considerably small to justify the simplification. Fig. 2 shows the dependence of the predictions on various four-layer models. The dependence range within $\pm 10^{\circ}$ was observed to be around their average value. Therefore, the importance of average value is signified, which considers all the possible choices using the Boltzmann weight based on the adsorption energy, $E_{\text {ads }}^{\text {water }}$, as a measure of the stability. The average value $\left(78.47^{\circ}\right)$, is similar to the experimental values $[25,26]$, owing to the higher contact angles predicted for smaller $N$-mers (monomer and dimer).

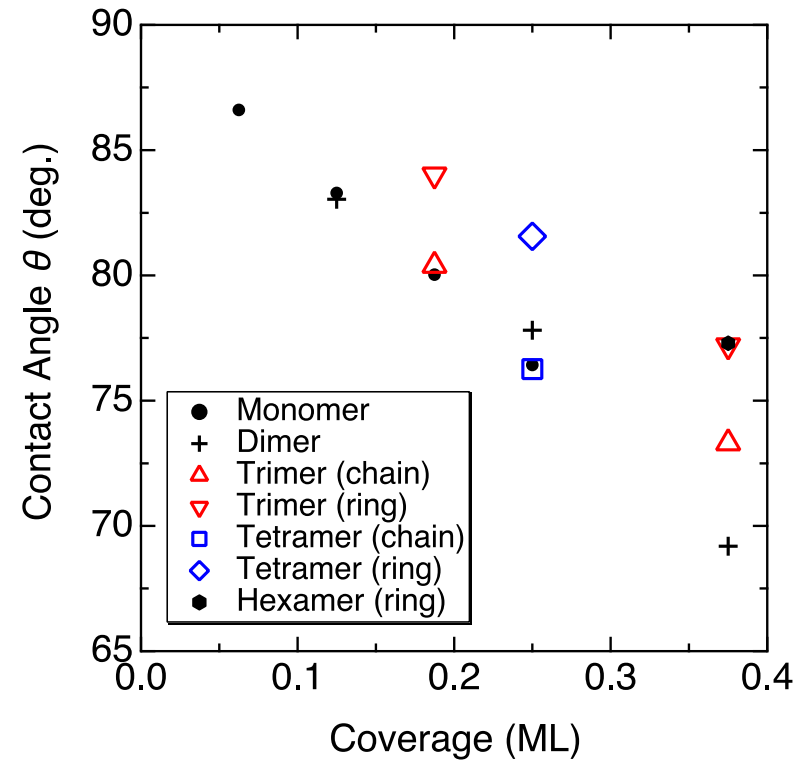

FIG. 2. Dependence of the predicted contact angles on various adsorption models for the adsorption of water molecules on the metallic $\mathrm{Cu}(111)$ surface.

Further, we examined the reliability of results by comparing the intermediate values obtained using Eq. (4), $\gamma_{\text {ice }}$, and $E_{\text {ads }}^{\text {water }}$, with those obtained in the previous studies. The surface energy value obtained in this study $\left(\gamma_{\text {ice }}=41.83\left[\mathrm{meV} / \AA^{2}\right]\right)$ is comparable to that reported in a previous DFT study (44 [meV/ $\left.\left.\AA^{2}\right]\right)$, [1] indicating a fair coincidence. In addition, the adsorption energy estimated in this study $\left(E_{\mathrm{ads}}^{\mathrm{watar}}=238\left[\mathrm{meV} / \mathrm{H}_{2} \mathrm{O}\right]\right.$ (monomer) $)$ is lower than that of the experimental value of $\mathrm{Cu}(111)\left(352\left[\mathrm{meV} / \mathrm{H}_{2} \mathrm{O}\right] \approx 34[\mathrm{~kJ} / \mathrm{mol}]\right)$ obtained using a temperature-programmed desorption evaluation (TPD). [21] The apparent underestimation of our value can be attributed to the fact that the experimental values consider the energy required to break the hydrogen bonds during the evaporation of water molecules, thereby resulting in the more stabilized value. The 
same explanation is applicable to the DFT values (for monomer) obtained in our study, which are smaller than those obtained in previous studies $\left(145-157\left[\mathrm{meV} / \mathrm{H}_{2} \mathrm{O}\right][20]\right.$ and $187\left[\mathrm{meV} / \mathrm{H}_{2} \mathrm{O}\right]$ $\approx 18[\mathrm{~kJ} / \mathrm{mol}])[21])$. In addition, the DFT values for dimers in previous studies $(321-332$ $\left[\mathrm{meV} / \mathrm{H}_{2} \mathrm{O}\right][20]$ and $\left.\left.352\left[\mathrm{meV} / \mathrm{H}_{2} \mathrm{O}\right] \approx 34[\mathrm{~kJ} / \mathrm{mol}]\right)[21]\right)$ were larger than those obtained in this study. The significant increase in values for the dimer can be attributed to the difference in definition. In their definition, the hydrogen bonding interactions between water molecules were included in the $E_{\mathrm{ads}}^{\mathrm{watar}}$, and hence, it increased with an increase in the size of $N$-mer.

\section{A. Choice of exchange-correlation potentials}

DFT predictions are known to critically depend on the choice of exchange-correlation potentials. [2, 3, 27] Because we are dealing with the adsorption of a monolayer of water molecules, chemisorption would be dominant, so we did not consider dispersion interactions [28] when employing GGA-PBE for the exchange-correlation potential. However, correcting for dispersion interactions $[29,30]$ may affect the present results. Another possible correction would be for spurious dipole interactions in the modeling of surfaces using a periodic slab structure. [31, 32] We thus evaluated the effects of both the dispersion and dipole corrections, as shown in Table III. Owing to the restrictions of CASTEP implementation, the available corrections are limited to those on GGA-PBE with norm-conserving pseudopotentials. For the dispersion corrections, we used TS [29] and Grimme [30] schemes, whereas for the dipole corrections, we applied NSC (nonself-consistent) [31] and SC (self-consistent) [32], as they are available in CASTEP. As shown

in Table III, the dispersion corrections lead to a larger magnitude of $E_{\text {ads }}^{\text {water }}$ as a consequence of the shorter bonding length to the surface. The dispersion correction is then found to reduce the contact angle by approximately $14^{\circ}$, whereas dipole corrections cause a smaller reduction of approximately $2^{\circ}$. When we applied both corrections, the contact angle is corrected by approximately $\delta \theta \sim-15^{\circ}$, thus deviating even further from the experimental value. However, the final conclusion of the present study, namely, that the isolated model is appropriate, would not be affected by these corrections. The reduction $\delta \theta$ is mainly due to the contraction of the bonding length, i.e., the interaction vertical to the surface, whereas the difference between the isolated and bilayer models lies in the intermolecular interactions in the horizontal directions on the surface. The vertical interactions are common to both isolated and bilayer models and hence do not change the conclusion that the isolated model provides values closer to the experimental ones. 
TABLE III. Comparisons of the predicted contact angle with and without dispersion correction (Disp.Corr.) and dipole correction (Dipole Corr.), estimated for the isolated-hexamer (nine-layer) model.

\begin{tabular}{cccc}
\hline Correction & Method & Contact angle $\left[{ }^{\circ}\right]$ Bonding length $[\AA]$ \\
\hline Without Corr. & - & 79.59 & 2.80 \\
\hline Disp. Corr. & TS & 65.12 & 2.50 \\
& Grimme & 66.80 & 2.36 \\
\hline Dipole Corr. & NSC & 76.90 & 2.77 \\
& SC & 77.35 & 2.75 \\
\hline Disp.+Dipole Corr. & TS/NSC & 62.91 & 2.47 \\
& TS/SC & 63.21 & 2.47 \\
& Grimme/NSC & 65.73 & 2.47 \\
& Grimme/SC & 66.11 & 2.46 \\
\hline
\end{tabular}

Because the valence shell of $\mathrm{Cu}$ consists of $d$-electrons, the Hubbard $U$ correction [33-35] may also be important. However, this correction matters when the orbital becomes more localized where the self-interaction cancellation is damaged. [36] For our metallic $\mathrm{Cu}$ surface, the orbital is delocalized, and hence, we did not consider such corrections, as in most preceding studies. [37]

\section{B. Structural model and the evaluation method}

Our conclusion that the isolated model is appropriate for the metallic $\mathrm{Cu}(111)$ surface contradicts the cases of $\operatorname{Pd}(111), \operatorname{Pt}(111)$, and $\operatorname{Ru}(0001)$ surfaces. [38, 39] For these surfaces, molecular coverage with horizontal intermolecular interactions is predicted to be the stable surface structure. This contrast can be attributed to the difference in coverage depending on the adsorption energy. As explained below, the coverage for $\mathrm{Cu}(111)$ becomes lower than those for $\mathrm{Pd}, \mathrm{Pt}$, and $\mathrm{Ru}$. Although the lower coverage for $\mathrm{Cu}$ is well described by the isolated model, higher coverage is better described by a network of molecules with interactions. The lower coverage is due to the weaker adsorption of molecules on $\mathrm{Cu}(111)$, namely, $352\left[\mathrm{meV} / \mathrm{H}_{2} \mathrm{O}\right]$, as estimated by TPD experiments, [21] which is approximately half of the theoretical estimations for Pd, Pt, and Ru. [38] The molecules adsorbed on $\mathrm{Cu}$ then more easily desorb again from the surface, leading to the lower coverage than those for $\mathrm{Pd}, \mathrm{Pt}$, and $\mathrm{Ru}$. This lower coverage was actually derived in a preceding 
study [40] by using a Langmuir adsorption isotherm. [41] The dependence on the adsorption energy appears through the adsorption equilibrium constant as a function of the Boltzmann factor. Another DFT estimation demonstrated a coverage of $~ 0.4 \mathrm{ML}$ above $250 \mathrm{~K}$, [40] which is closer to that of isolated model $[6 / 16(=0.375) \mathrm{ML}]$ than that of the bilayer model $[6 / 9(\sim 0.667) \mathrm{ML}]$. Corresponding to the lower coverage, isolated monomers, dimers, trimers, and tetramers have actually been observed in STM (scanning tunneling microscope) experiments on several metallic surfaces. [14]

Because the Langmuir adsorption isotherm [41] is basically applicable to solid-gas (steam) interfaces, applying it to a solid-liquid interface requires careful consideration. Applying it in this case would be justified when (i) the intermolecular interactions between water molecules are negligible, and (ii) the absorption process of the water molecules on the solid surface is equilibrated and then none of the absorbed molecules are dissociated into $\mathrm{H}^{+}$and $\mathrm{OH}^{-}$, so the molecules in the liquid water can then be regarded as molecules in steam. The isolated model satisfies (i), and the resultant lower coverage seems self-consistent. For (ii), the dissociation energy of the water molecules on $\mathrm{Cu}(111)$ has been reported to be $1.23\left[\mathrm{eV} / \mathrm{H}_{2} \mathrm{O}\right]$, [42] being much larger than the energy scale of the adsorption energy, $\sim 352\left[\mathrm{meV} / \mathrm{H}_{2} \mathrm{O}\right]$. [21] A previous DFT study [40] also concluded that the adsorption of water molecules on $\mathrm{Cu}(111)$ without dissociation is much more stable than that with dissociation.

In Eq. (2) of the present study, the left-hand side denotes the balance of forces horizontal to the surface acting as the boundary of the liquid-covered region. By achieving coverage, the system becomes stabilized by $E_{\text {ads }}$, as indicated by the right-hand side of the equation. However, not only this stabilization term but also another term, $\gamma_{\mathrm{intMol}}$, contributes to pulling the boundary inside toward liquid-covered region owing to intermolecular interactions. This tension term can be omitted when the intermolecular distance increases under the lower coverage, but when the coverage increases, the approximation in Eq. (2) makes the the contact angle less accurate. To justify using this equation in prior studies, $[1,11,43]$ the structural models had to be "sufficiently sparse" to omit $\gamma_{\text {intMol }}$. Because our isolated model is more sparse than the honeycomb models in those studies, the approximation in Eq. (2) is even more reliable in the present work. Furthermore, using GGA-PBE without considering intermolecular interactions is more consistent with the omission of $\gamma_{\mathrm{intMol}}$. If we include the dispersion correction, $\gamma_{\mathrm{intMol}}$ would become more pronounced, which would increase the contact angle solely by pulling the boundary inside the wet region. The results 
shown in Table III, however, are the opposite, i.e., considering the dispersion correction actually reduces the angle. This reduction is caused by another mechanism, as explained above, i.e., the shortening of the vertical bond to the surface. At least within the exchange-correlation potential we used, the horizontal dispersion interactions between molecules thus seem smaller than those for the vertical molecular-surface binding. This is also consistent with omitting $\gamma_{\text {intMol }}$ from Eq. (2).

\section{Comparison with other methodologies}

Herein, we compare the computational evaluations of the contact angle obtained herein with those obtained previously because it will be helpful for deeper understanding of the proposed scheme. The computational evaluations of the contact angle are mainly classified into two schemes: (I) energetics combined with Young's relation in Eq. (1) (indirect approach) and (II) direct observations of droplet geometry (direct approach). [44-46] Based on the scheme, several choices of interface modeling and computational methodologies are possible. Hereafter, we discuss the universality of each approach.

(I) The indirect approach first evaluates interface free energies by any means and then computes the contact angle via Eq. (1). The free energies can be (approximately) evaluated from various simulations including not only ab initio [electronic level] but also molecular dynamics (MD) and/or Monte Carlo (MC) [molecular level]. The accuracy of the contact angle depends on the adopted interface modeling and level of theory.

The $a b$ initio simulations with interface modeling appropriate for insulating surfaces ("ice-like bilayer" model) have proven to accurately reproduce the contact angles. $[1,11]$ Furthermore, the present study demonstrates the applicability of the $a b$ initio approach to metallic surfaces. As explained in the present study, our interface modeling ("isolated cluster" model) relies on the experimental facts about how water clusters are absorbed into surfaces. Because water clusters on various metallic surfaces have been investigated comprehensively [47], the interface modeling appropriate for individual metallic surfaces can be achieved without any difficulties. Although the accuracy of $a b$ initio DFT energies generally depend on the exchange-correlation function, but as far as the water absorption on metallic surfaces is concerned, DFT is expected to work efficiently.

As for the molecular levels, several combinations of interface modeling and the molecular simulations have been developed. Within the framework of the molecular simulation, the interface energy is thermodynamically described via fundamental quantities that can be evaluated by 
MD/MC sampling. The phantom-wall method [48, 49], dry-surface method [50], and interface wetting potential method [51,52] are combined with MD simulations; the test-area perturbation approach [53,54] and the excess surface free energy approach [55-57] employ MC simulations. As explained later, $\mathrm{MD} / \mathrm{MC}$ requires molecular force fields appropriate for individual metallic surfaces; therefore, its applicability strongly depends on available force fields.

(II) The direct approach usually employs MD-based simulations to construct molecular droplets and attempts to gauge the contact angles from the MD snapshots of the droplet geometries. Several schemes $[44,58-60]$ have been proposed to identify the "droplet shape" from the molecular condensation; then, the contact angle is evaluated via elemental differential geometry. Note that this direct approach (II) involves more uncertainty than the indirect one (I), but the direct one is applicable even for determining wettability on rough surfaces and dynamic wettability. This implies that (i) the indirect approach provides more accurate (static) contact angle estimations than the direct one, assuming the interface energies to be accurate [46], and (ii) the direct approach provides more general estimations applicable to a wide range of wettability phenomena [44].

In both the direct and indirect approaches, the accurate prediction of the contact angle strongly depend on that of molecular interactions at each interface. Molecular force fields used in MD/MC simulations are crucial for reproducing the molecular interactions [61], whereas ab initio DFT approaches usually predict the energies accurately. In MD simulations, Lennard-Jones (LJ) potentials associated with electrostatic ones are mostly selected as the force field. [62] The LJ potentials require a set of parameters for each molecular interaction; major parameter sets are available for water $[62,63]$, but individual parameter fittings are necessary for different surfaces by comparing with $a b$ initio simulations or experiments. $[64,65]$ MD simulations with various types of the force fields have been applied to evaluate water contact angles on metallic surfaces such as Pt [66] and face-centered cubic $\mathrm{Cu}$ [67], but the accuracy of their predictions has been reported to vary depending on the force fields adopted. [59]

Recently, several attempts have been made to address the force field issue in classical MD. A straightforward way is to directly improve the force field accuracy by fitting DFT data, [68] although its prediction should be calibrated by the experimental validation. [59] Further, ab initio MD (AIMD) has been applied to surface/interface properties of water and evaluated the surface tension [69] and the contact angle [70]. The AIMD overcomes (semi)empirical force fields, but has a remarkable limitation on system sizes; hundreds of water molecules in a previous study [70] were insufficient to identify the "droplet shape," as more than 500k water molecules were required 
for that purpose [60]. Within the framework of the direct approach, ab initio evaluations of the contact angle are still a challenge for AIMD, whereas classical MD involves a number of caseby-case parameter fittings. From the viewpoint of complexity of simulation procedures, our DFTbased indirect approach can be regarded as being simpler than the MD-based direct approach.

Finally, we consider the temperature dependence of the contact angles. For the water wetting, its contact angles at ambient/low temperatures (less than a boiling point) remain almost constant, whereas those at high temperatures suddenly decrease. [71] For example, the contact angles of water on an aluminum surface are independent of the temperature at $20<T<120^{\circ} \mathrm{C}$ being $90^{\circ}$, while the temperature dependence is given as $157.4-0.55 T$ at $T \geq 120^{\circ} \mathrm{C}$. Looking at our scheme, we took the Boltzmann averaging over several types of water clusters to evaluate the contact angle, which might be expected to describe the temperature dependence. However, our scheme provides almost constant values of the contact angles within $0.2^{\circ}$ for a wide range of temperatures $0<T<150^{\circ} \mathrm{C}$. These values are consistent with those at low temperatures, whereas they are inconsistent with those at high temperatures. This is because no evaporation is assumed in our scheme, while in reality, water evaporates at high temperatures, thereby reducing the surface tension as the temperature increases. $[63,72]$ Because our scheme assumes that there is no intake/outtake of water molecules, the applicability of our scheme is restricted to low/ambient temperature regions. We thus conclude that our scheme would successfully predict the contact angles at low temperatures.

\section{Limitations in comparison with reality}

We note that the comparison with experiments shown in Table II should be made only for the limited purpose of demonstrating the superiority of our structural model over the conventional honeycomb model. $[13,14]$ Though the present model achieves the maximum possible toward the reality within the available computational feasibility, there are several unsatisfactory factors to describe the reality as we can point out below. To obtain a quantitatively reliable estimate for the interaction energy between water and the surface, one would wonder that the larger coverages than one bilayer as well as structural disorder in the water layer should be considered. To capture such factors, the larger simulation cells are required. The feasibility of practical ab initio calculations is, however, subject to limitations on simulation sizes. The computational cost typically scales as the cube of the system size. [73] The available memory capacity is far smaller than that required 
to store the dynamic updates of such a larger system.

Not only in the present model, but also in all the current $a b$ initio treatments, $\gamma_{L G}$ is approximately modelled by the surface energy of $I h$-ice. $[1,11]$ Though there are several possible criticisms against this approximations, this modelling is actually the keystone for the treatment, otherwise it becomes infeasible to describe the contact angle in the ab initio framework within the practical computational cost. At least within the current feasibility, we can either (a) compromise between the limitation of the modeling and the universality of $a b$ initio framework to capture the trends with the same formalism, or (b) abandon the universality, just focusing on a specific system to pursue the descriptiveness of reality by using qualitative model-theoretical treatments.

As we discussed in Table III, the dependence of the predictions on the exchange-correlation functionals is another point for further investigations. The functionals capable of capturing the dispersion interactions have gradually be underway, but they are typically 10 times heavier than the conventional ones in their computational cost as a result of the extra processing (e.g., nonlocality) required. [74] Hence, from the viewpoint of the computational cost, it is a trade-off between the direction toward capturing the disorder effects and that toward describing the dispersion interactions in detail.

It should be noted, again, that the possible criticism on the incompleteness of the modeling enumerated above is not specific to the present modeling, but is commonly applied to all the previous $a b$ initio studies. Although the common criticism still remains unsolved, the superiority over the conventional geometrical modeling has been performed for the metallic surfaces within the available computational resources at the best effort, as emphasized again.

\section{CONCLUSIONS}

In this study, an ab initio evaluation of the water contact angle on $\mathrm{Cu}(111)$ surface was performed by determining the surface energy of water $\left(\sim \gamma_{\mathrm{LG}}\right)$ using that of ice crystal, which has been widely used in previous studies. $[1,11]$ The results revealed that the isolated water oligomers used in this study as the adsorption model afforded a more accurate prediction of the water contact angle on the metallic $\mathrm{Cu}(111)$ surface than that obtained by the periodic-honeycomb lattice of ice crystals, which has been widely used for estimating the water contact angle on the surfaces of insulating materials. $[1,11]$ The results were supported by several experimental observations. $[14,16,21]$ For the freedom of the model choice (namely, the size of the oligomers and the 
coverage), we revealed that averaging out the possible choices using the Boltzmann weight based on the adsorption energies leads to a fairly reasonable prediction compared with the experimental values.

\section{ACKNOWLEDGMENTS}

The computations in this work were performed using the facilities of Research Center for Advanced Computing Infrastructure at JAIST. K.H. is grateful for financial support from the HPCI System Research Project (Project ID: hp190169 and hp200040) and MEXT-KAKENHI (JP16H06439, JP17K17762, JP19K05029, and JP19H05169). R.M. is grateful for financial support from MEXT-KAKENHI (JP19H04692 and JP16KK0097), FLAGSHIP2020 (project nos. hp190169 and hp190167 at K-computer), Toyota Motor Corporation, the Air Force Office of Scientific Research (AFOSR-AOARD/FA2386-17-1-4049;FA2386-19-1-4015), and JSPS Bilateral Joint Research Projects (with India DST).

\section{AUTHOR INFORMATION}

\section{A. Contributions}

K.H. conceived this work. K.H. and R.M. directed and supervised this work. T.M. performed the computations and acquired the computational data. T.M. interpreted the computational data and wrote the first draft of the manuscript. The manuscript was written with significant contributions from all authors.

\section{B. Correponding author}

Correspondence to Kenta Hongo or Takahiro Murono. 


\section{REFERENCES}

[1] Giuliano Carchini, Max García-Melchor, Zbigniew Łodziana, and Núria López, "Understanding and Tuning the Intrinsic Hydrophobicity of Rare-Earth Oxides: A DFT+U Study," ACS Appl. Mater. Interfaces 8, 152-160 (2016).

[2] Kenta Hongo and Ryo Maezono, "A Computational Scheme To Evaluate Hamaker Constants of Molecules with Practical Size and Anisotropy,” J. Chem. Theory Comput. 13, 5217-5230 (2017).

[3] Hideyuki Takagishi, Takashi Masuda, Tatsuya Shimoda, Ryo Maezono, and Kenta Hongo, "Method for the Calculation of the Hamaker Constants of Organic Materials by the Lifshitz Macroscopic Approach with Density Functional Theory," J. Phys. Chem. A 123, 8726-8733 (2019).

[4] Patricia A Thiel and Theodore E Madey, "The interaction of water with solid surfaces: Fundamental aspects," Surf. Sci. Rep. 7, 211-385 (1987).

[5] Michael A Henderson, "The interaction of water with solid surfaces: fundamental aspects revisited," Surf. Sci. Rep. 46, 1-308 (2002).

[6] Kelsey A. Stoerzinger, Wesley T. Hong, Gisele Azimi, Livia Giordano, Yueh Lin Lee, Ethan J. Crumlin, Michael D. Biegalski, Hendrik Bluhm, Kripa K. Varanasi, and Yang Shao-Horn, "Reactivity of Perovskites with Water: Role of Hydroxylation in Wetting and Implications for Oxygen Electrocatalysis,” J. Phys. Chem. C 119, 18504-18512 (2015).

[7] Y. Yuan and T. R. Lee, Springer Series in Surface Sciences, Vol. 51 (Springer, Berlin, Heidelberg, 2013) pp. 3-34.

[8] Tommi Huhtamäki, Xuelin Tian, Juuso T. Korhonen, and Robin H. A. Ras, "Surface-wetting characterization using contact-angle measurements," Nat. Protoc. 13, 1521-1538 (2018).

[9] Thomas Young, "III. An essay on the cohesion of fluids," Phil. Trans. R. Soc. 95, 65-87 (1805).

[10] P. G. de Gennes, “Wetting: statics and dynamics," Rev. Mod. Phys. 57, 827-863 (1985).

[11] B. Lange, R. Posner, K. Pohl, C. Thierfelder, G. Grundmeier, S. Blankenburg, and W. G. Schmidt, "Water adsorption on hydrogenated Si(1 11 1) surfaces," Surf. Sci. 603, 60-64 (2009).

[12] C. J. Van Oss, R. F. Giese, R. Wentzek, J. Norris, and E. M. Chuvilin, "Surface Tension Parameters of Ice Obtained From Contact Angle Data and From Positive and Negative Particle Adhesion to Advancing Freezing Fronts,” J. Adhes. Sci. Technol. 6, 503-516 (1992). 
[13] A. Hodgson and S. Haq, "Water adsorption and the wetting of metal surfaces," Surf. Sci. Rep. 64, $381-451$ (2009).

[14] Javier Carrasco, Andrew Hodgson, and Angelos Michaelides, "A molecular perspective of water at metal interfaces," Nat. Mater. 11, 667-674 (2012).

[15] Karina Morgenstern and Karl Heinz Rieder, "Formation of the cyclic ice hexamer via excitation of vibrational molecular modes by the scanning tunneling microscope," J. Chem. Phys. 116, 5746-5752 (2002).

[16] Angelos Michaelides and Karina Morgenstern, "Ice nanoclusters at hydrophobic metal surfaces," Nat. Mater. 6, 597-601 (2007).

[17] Stewart J. Clark, Matthew D. Segall, Chris J. Pickard, Phil J. Hasnip, Matt I.J. Probert, Keith Refson, and Mike C. Payne, "First principles methods using CASTEP,” Z. Kristallogr. 220, 567-570 (2005).

[18] John P. Perdew, Kieron Burke, and Matthias Ernzerhof, "Generalized gradient approximation made simple,” Phys. Rev. Lett. 77, 3865-3868 (1996).

[19] D. R. Hamann, M. Schlüter, and C. Chiang, "Norm-Conserving Pseudopotentials," Phys. Rev. Lett. 43, 1494-1497 (1979).

[20] Angelos Michaelides, "Simulating ice nucleation, one molecule at a time, with the 'DFT microscope'," Faraday Discuss. 136, 287-297 (2007).

[21] Qian Lin Tang and Zhao Xu Chen, "Density functional slab model studies of water adsorption on flat and stepped Cu surfaces," Surf. Sci. 601, 954-964 (2007).

[22] Hendrik J. Monkhorst and James D. Pack, “Special points for brillouin-zone integrations,” Phys. Rev. B 13, 5188-5192 (1976).

[23] Kathleen Lonsdale, “The structure of ice,” Proc. R. Soc. Lond. A 247, 424-434 (1958).

[24] R. Chidambaram, "A bent hydrogen bond model for the structure of ice-I," Acta Cryst. 14, 467-468 (1961).

[25] Jimin Park, Hyung Seop Han, Yu Chan Kim, Jae Pyeong Ahn, Myoung Ryul Ok, Kyung Eun Lee, Jee Wook Lee, Pil Ryung Cha, Hyun Kwang Seok, and Hojeong Jeon, "Direct and accurate measurement of size dependent wetting behaviors for sessile water droplets," Sci. Rep. 5, 18150 (2015).

[26] M. Voinea, C. Vladuta, C. Bogatu, and A. Duta, "Surface properties of copper based cermet materials," Mater. Sci. Eng. B 152, 76-80 (2008).

[27] Kenta Hongo, Nguyen Thanh Cuong, and Ryo Maezono, "The Importance of Electron Correlation on Stacking Interaction of Adenine-Thymine Base-Pair Step in B-DNA: A Quantum Monte Carlo Study,” 
J. Chem. Theory Comput. 9, 1081-1086 (2013).

[28] Alston J. Misquitta, Ryo Maezono, Neil D. Drummond, Anthony J. Stone, and Richard J. Needs, “Anomalous nonadditive dispersion interactions in systems of three one-dimensional wires," Phys. Rev. B 89, 045140 (2014).

[29] Alexandre Tkatchenko and Matthias Scheffler, "Accurate molecular van der Waals interactions from ground-state electron density and free-atom reference data," Phys. Rev. Lett. 102, 6-9 (2009).

[30] Stefan Grimme, "Semiempirical GGA-Type Density Functional Constructed with a Long-Range Dispersion Correction,” J. Comput. Chem. 27, 1787-1799 (2006).

[31] In Chul Yeh and Max L. Berkowitz, "Ewald summation for systems with slab geometry," J. Chem. Phys. 111, 3155-3162 (1999).

[32] Jorg Neugebauer and Matthias Scheffler, "Adsorbate-substrate and adsorbate-adsorbate interactions of Na and K adlayers on Al(111),” Phys. Rev. B 46, 16067-16080 (1992).

[33] Adie Tri Hanindriyo, Soumya Sridar, K.C. Hari Kumar, Kenta Hongo, and Ryo Maezono, “Ab initio thermodynamic properties of certain compounds in nd-fe-b system," Comput. Mater. Sci. 180, 109696 (2020).

[34] Tom Ichibha, Anouar Benali, Kenta Hongo, and Ryo Maezono, "Ti interstitial flows giving rutile tio 2 reoxidation process enhancement in (001) surface,” Phys. Rev. Materials 3, 125801 (2019).

[35] S Khajoei Gharaei, M Abbasnejad, and Ryo Maezono, "Bandgap reduction of photocatalytic TiO2 nanotube by Cu doping," Scientific Reports 8, 14192 (2018).

[36] Vladimir I. Anisimov, Jan Zaanen, and Ole K. Andersen, "Band theory and mott insulators: Hubbard u instead of stoner i,” Phys. Rev. B 44, 943-954 (1991).

[37] Takamasa Makino, Michio Okada, and Anton Kokalj, "Adsorption of c2h4 on stepped cu(410) surface: A combined tpd, ftir, and dft study," J. Phys. Chem. C 118, 27436-27448 (2014), https://doi.org/10.1021/jp509228v.

[38] Guillem Revilla-López and Núria López, “A unified study for water adsorption on metals: meaningful models from structural motifs," Phys. Chem. Chem. Phys. 16, 18933-18940 (2014).

[39] Luca Bellarosa, Rodrigo García-Muelas, Guillem Revilla-López, and Núria López, "Diversity at the Water-Metal Interface: Metal, Water Thickness, and Confinement Effects,” ACS Cent. Sci. 2, 109-116 (2016).

[40] Beien Zhu, Zhen Xu, Chunlei Wang, and Yi Gao, "Shape Evolution of Metal Nanoparticles in Water Vapor Environment," Nano Lett. 16, 2628-2632 (2016). 
[41] Irving Langmuir, “THE ADSORPTION OF GASES ON PLANE SURFACES OF GLASS, MICA AND PLATINUM.” J. Am. Chem. Soc. 40, 1361-1403 (1918).

[42] Gui-Chang Wang and Junji Nakamura, "Structure Sensitivity for Forward and Reverse Water-Gas Shift Reactions on Copper Surfaces: A DFT Study,” J. Phys. Chem. Lett. 1, 3053-3057 (2010).

[43] Georgios D. Barmparis and Ioannis N. Remediakis, "Dependence on co adsorption of the shapes of multifaceted gold nanoparticles: A density functional theory," Phys. Rev. B 86, 085457 (2012).

[44] Srikanth Ravipati, Benjamin Aymard, Serafim Kalliadasis, and Amparo Galindo, "On the equilibrium contact angle of sessile liquid drops from molecular dynamics simulations,” J. Chem. Phys. 148, 164704 (2018).

[45] Ilham Essafri, Jean Christophe Le breton, Arnaud Saint-Jalmes, Armand Soldera, Anthony Szymczyk, Patrice Malfreyt, and Aziz Ghoufi, "Contact angle and surface tension of water on a hexagonal boron nitride monolayer: a methodological investigation,” Mol. Simul. 45, 454-461 (2019).

[46] Hao Jiang and Amish J. Patel, "Recent advances in estimating contact angles using molecular simulations and enhanced sampling methods," Curr. Opin. Chem. Eng. 23, 130-137 (2019).

[47] Tomoko K. Shimizu, Sabine Maier, Albert Verdaguer, Juan-Jesus Velasco-Velez, and Miquel Salmeron, "Water at surfaces and interfaces: From molecules to ice and bulk liquid," Prog. Surf. Sci. 93, 87-107 (2018).

[48] Frédéric Leroy, Daniel J.V.A. dos Santos, and Florian Müller-Plathe, "Interfacial excess free energies of solid-liquid interfaces by molecular dynamics simulation and thermodynamic integration," Macromol. Rapid Commun. 30, 864-870 (2009).

[49] Frédéric Leroy and Florian Müller-Plathe, "Solid-liquid surface free energy of Lennard-Jones liquid on smooth and rough surfaces computed by molecular dynamics using the phantom-wall method," J. Chem. Phys. 133, 044110 (2010).

[50] Frédéric Leroy and Florian Müller-Plathe, "Dry-Surface Simulation Method for the Determination of the Work of Adhesion of Solid-Liquid Interfaces," Langmuir 31, 8335-8345 (2015).

[51] Matej Kanduč and Roland R. Netz, "From hydration repulsion to dry adhesion between asymmetric hydrophilic and hydrophobic surfaces," Proc. Natl. Acad. Sci. U.S.A. 112, 12338-12343 (2015).

[52] Matej Kanduč and Roland R. Netz, "Atomistic simulations of wetting properties and water films on hydrophilic surfaces," J. Chem. Phys. 146, 164705 (2017).

[53] Guy J. Gloor, George Jackson, Felipe J. Blas, and Enrique de Miguel, “Test-area simulation method for the direct determination of the interfacial tension of systems with continuous or discontinuous 
potentials,” J. Chem. Phys. 123, 134703 (2005).

[54] C. Ibergay, A. Ghoufi, F. Goujon, P. Ungerer, A. Boutin, B. Rousseau, and P. Malfreyt, "Molecular simulations of the $n$-alkane liquid-vapor interface: Interfacial properties and their long range corrections," Phys. Rev. E 75, 051602 (2007).

[55] Eric M. Grzelak and Jeffrey R. Errington, "Computation of interfacial properties via grand canonical transition matrix Monte Carlo simulation,” J. Chem. Phys. 128, 014710 (2008).

[56] Kaustubh S. Rane, Vaibhaw Kumar, and Jeffrey R. Errington, "Monte Carlo simulation methods for computing the wetting and drying properties of model systems," J. Chem. Phys. 135, 234102 (2011).

[57] Vaibhaw Kumar and Jeffrey R. Errington, "The use of Monte Carlo simulation to obtain the wetting properties of water," Phys. Procedia 53, 44-49 (2014).

[58] M. J. de Ruijter, T. D. Blake, and J. De Coninck, "Dynamic wetting studied by molecular modeling simulations of droplet spreading," Langmuir 15, 7836-7847 (1999).

[59] T Werder, J H Walther, R L Jaffe, T Halicioglu, and P Koumoutsakos, "On the Water-Carbon Interaction for Use in Molecular Dynamics Simulations of Graphite and Carbon Nanotubes," J. Phys. Chem. B 107, 1345-1352 (2003).

[60] Erik E. Santiso, Carmelo Herdes, and Erich A. Müller, "On the calculation of solid-fluid contact angles from molecular dynamics," Entropy 15, 3734-3745 (2013).

[61] Fereshte Taherian, Valentina Marcon, Nico F.A. van der Vegt, and Frédéric Leroy, "What is the contact angle of water on graphene?” Langmuir 29, 1457-1465 (2013).

[62] Alessio Alexiadis and Stavros Kassinos, "Molecular simulation of water in carbon nanotubes," Chem. Rev. 108, 5014-5034 (2008).

[63] C. Vega, J. L. F. Abascal, M. M. Conde, and J. L. Aragones, "What ice can teach us about water interactions: a critical comparison of the performance of different water models," Faraday Discuss. 141, 251-276 (2009).

[64] Sheng Bai Zhu and Michael R. Philpott, "Interaction of water with metal surfaces," J. Chem. Phys. 100, 6961-6968 (1994).

[65] Hendrik Heinz, R. A. Vaia, B. L. Farmer, and R. R. Naik, "Accurate simulation of surfaces and interfaces of face-centered cubic metals using 12-6 and 9-6 lennard-jones potentials," J. Phys. Chem. C 112, 17281-17290 (2008).

[66] Bo Shi and Vijay K. Dhir, "Molecular dynamics simulation of the contact angle of liquids on solid surfaces," J. Chem. Phys. 130, 034705 (2009). 
[67] W. Xu, Z. Lan, B. L. Peng, R. F. Wen, and X. H. Ma, "Effect of surface free energies on the heterogeneous nucleation of water droplet: A molecular dynamics simulation approach,” J. Chem. Phys. 142, $054701(2015)$.

[68] Yanbin Wu and N. R. Aluru, "Graphitic carbon-water nonbonded interaction parameters," J. Phys. Chem. B 117, 8802-8813 (2013).

[69] Yuki Nagata, Tatsuhiko Ohto, Mischa Bonn, and Thomas D. Kühne, "Surface tension of ab initio liquid water at the water-air interface,” J. Chem. Phys. 144, 204705 (2016).

[70] Hui Li and Xiao Cheng Zeng, "Wetting and interfacial properties of water nanodroplets in contact with graphene and monolayer boron-nitride sheets," ACS Nano 6, 2401-2409 (2012).

[71] John D. Bernardin, Issam Mudawar, Christopher B. Walsh, and Elias I. Franses, "Contact angle temperature dependence for water droplets on practical aluminum surfaces,” Int. J. Heat Mass Transfer 40, 1017 - 1033 (1997).

[72] C. Vega and E. de Miguel, "Surface tension of the most popular models of water by using the test-area simulation method," J. Chem. Phys. 126, 154707 (2007), https://doi.org/10.1063/1.2715577.

[73] Matúš Dubecký, Lubos Mitas, and Petr Jurečka, "Noncovalent Interactions by Quantum Monte Carlo," Chem. Rev. 116, 5188-5215 (2016).

[74] Jií Klimeš and Angelos Michaelides, "Perspective: Advances and challenges in treating van der Waals dispersion forces in density functional theory,” J. Chem. Phys. 137, 120901 (2012), 1301.6960. 


\section{Supplementary Files}

This is a list of supplementary files associated with this preprint. Click to download.

- si.pdf

- figS1icelikebilayeronCu1119layers.cif

- figS21H2OhexamercyclicbuckledonCu1119layers.cif

- figS34H2OmonomersonCu1114layers.cif

- figS81H2OtetramercycliconCu1114layers.cif

- figS52H2OtrimerschainonCu1114layers.cif

- figS91H2OhexamercyclicplanaronCu1114layers.cif

- figS43H2OdimersonCu1114layers.cif

- figS71H2OtetramerchainonCu1114layers.cif

- figS62H2OtrimerscycliconCu1114layers.cif 